\title{
Gut microbiome, surgical complications and probiotics
}

\section{George Stavrou, Katerina Kotzampassi}

Aristotle University of Thessaloniki, Thessaloniki, Greece

\section{Abstract}

\section{Introduction}

Complications after colorectal surgery - especially those performed for malignancy - are often a result of bacterial infections, leading to a significant increase in morbidity and mortality, as well as the duration of hospitalization and the subsequent costs. In this process, the gut seems to play a crucial part. Failure of the gut barrier function has long been considered to lead to a process called "bacterial translocation", where whole bacteria or their virulent products enter the systemic circulation and provoke systemic inflammatory response syndrome (SIRS), which may lead to multiple organ failure or even death. Human studies have shown that at least $11 \%$ of individuals who undergo an open-abdomen surgical operation have experienced translocation of live bacteria to the mesenteric lymph nodes or to the serosa of the bowel wall. Evidence of bacterial DNA in the blood of approximately $50 \%$ of

Department of Surgery, Faculty of Medicine, Aristotle University of Thessaloniki, Thessaloniki, Greece

Conflict of Interest: None

Correspondence to: Katerina Kotzampassi, MD, PhD, Department of Surgery, Aristotle University of Thessaloniki, Faculty of Medicine, Thessaloniki, Greece, e-mail: kakothe@yahoo.com

Received 05 May 2016; accepted 11 July 2016; published online 6 September 2016

DOI: http://dx.doi.org/10.20524/aog.2016.0086 patients in the intensive care unit (ICU) also suggests bacterial translocation, but there is still a great deal of controversy as to whether this is only an epiphenomenon, or whether it really contributes to morbidity [1,2].

In recent years, there has been ongoing interest in the human gut microbial ecosystem, which ultimately appears to be involved in both disease onset and progression, as well as in the development of complications. Moreover, there is increasing recognition of the important fact that microbes can obtain information from their host environment, which they then utilize to determine whether to colonize or express a virulent phenotype to invade the host, a scenario especially prevalent during prolonged critical illness [3-5].

In this review, efforts were made to present the newest data on the interrelationship between gut microbiome and the emergence of complications after colon surgery, and the efficacy of probiotics as therapeutic instruments for changing the bacterial imbalance.

\section{Intestinal microbiota: symbiosis and dysbiosis}

The gastrointestinal tract hosts a particularly complex microbial ecosystem, consisting of more than $10^{14}$ microbes representing 500-1500 species. This ecosystem remains relatively stable throughout life, leading to the speculation that individuals might possess a unique microbial "fingerprint", despite daily variations attributable to diet, lifestyle, age, 
and the host's physiological and immunological health. All microorganisms residing within or on the human body are called microbiota, and their genomes are known as the human microbiome [6,7].

The four dominant phyla inhabitants of the human gut are Firmicutes and Bacteroidetes, accounting for more than $90 \%$ of the bacteria cells, with a smaller representation of Actinobacteria and Proteobacteria. Species from the genus Bacteroides alone constitute about $30 \%$ of all bacteria in the human microbiome, while the well-known family Enterobacteriaceae, which contains medically relevant genera such as Escherichia, Klebsiella, Pseudomonas, and Salmonella, actually represents less than $1 \%$ [4,8-11] (Fig. 1).

This complex ecosystem coexists in a fragile balance (symbiosis), that can easily be disturbed (dysbiosis). This occurs when a disturbance in the composition and function of beneficial bacteria makes them incapable of controlling the harmful bacteria successfully. Today, dysbiosis has been linked with important human diseases, not only infections, but also autoimmune and autoinflammatory disorders, $[8,12]$. In this context, there is now clear evidence that every direct or indirect manipulation of gut microbiota - by means, for example, of antibiotics or surgery - contributes to disease development or the opposite: a broad range of medical and surgical problems are linked to perturbations of the microbiome (Table 1).

\section{Intestinal microbiome and colon surgery}

Intestinal microbiota and the human gut epithelium, serving as the host, maintain a long-term, well-tolerated symbiotic relationship. When the host "alters" the conditions of "hospitality", as occurs with the physiologic changes in the human body caused by surgical stress, and more specifically of the intestinal microenvironment, a disturbance in ecological balance occurs [13].

However, the fact that most surgical patients do not experience infectious complications simply underlines the adaptability of both the host and microbe in response to surgical stress $[1,4,14]$. It is also recognized that, besides the extent and severity of surgical stress, the variability of the inflammatory response is also mediated by genetic predisposition, the presence of comorbidities and the sideeffects of pharmacologic treatments.

In a recent study in piglets, DNA sequencing of the colonic content was studied comparatively in the "transection surgery" group and in the "no-surgery" group, two weeks after operation. Changes in the relative abundance of bacterial species were confined to Proteobacteria and Bacteroidetes phyla, while, at family level, there was evidence of a reduction in Enterobacteriaceae, Bacteroidaceae, and Rhodospirillaceae versus controls [15].

In colon surgery patients, there is not only the operative stress itself, but also a variety of perioperative interventions imposed by modern intensive care therapy, including preoperative bowel cleansing, multiple antibiotic exposure, prolonged starvation, exclusively intravenous nutrition, the administration of vasoactive agents, inhibitors of gastric

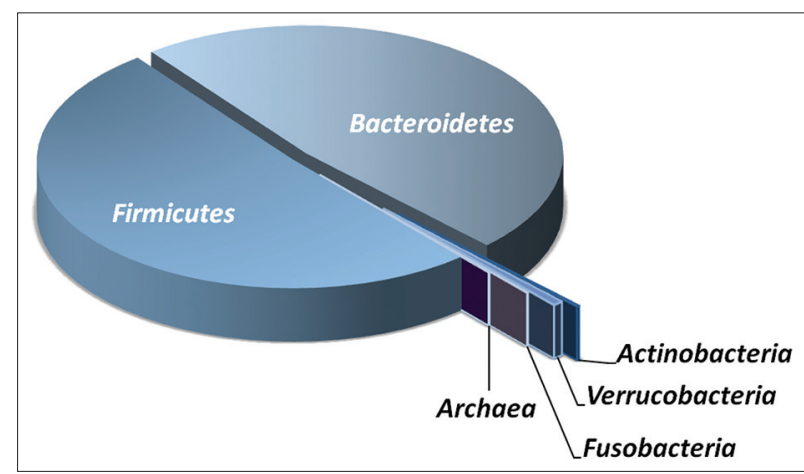

Figure 1 Distribution of the intestinal microbiota phyla

Table 1 Iatrogenic factors affecting the gut microbiome

Perioperative manipulations affecting gut microbiome

Mechanical bowel cleansing

Antibiotics

Stress-released hormones (catecholamines)

Vasoactive drugs (norepinephrine)

Endogenous and exogenous opioids (morphine)

Enteral feeding restriction

Micronutrients insufficiency

Operation/gut manipulation/resection/anastomosis

acidity, and opioids; and finally, the intense manipulation of the gut, which could disrupt the host-microbe relationship and thus could yield heightened virulence expression by bacteria and a fulminant inflammatory response in the host [1,15-18].

\section{Intestinal microbiome and mechanical bowel cleansing}

Mechanical bowel preparation for colorectal surgery has been normal routine for surgeons for more than a century; however, the Cochrane Database of Systematic Reviews, in an analysis of 18 trials with 5805 participants aimed at determining the safety and effectiveness of this preparation on morbidity and mortality in colorectal surgery patients, concluded that bowel cleansing can be safely omitted, as it is considered not to reduce rates of surgical site infections, unless it is combined with both oral and systemic antibiotics $[17,19]$.

By approaching the issue from the perspective of gut bacteria, a randomized controlled trial evaluated the effect of preoperative mechanical bowel cleansing on the fecal flora of patients undergoing colorectal surgery. They found a significant reduction in the total number of bacteria: Clostridium coccoides group, Clostridium leptum subgroup, Bifidobacteria, total Lactobacillus and Enterobacteriaceae were found to be significantly reduced, but there was no effect on the number of Enterococci and Staphylococci [16]. Similarly, from the 16S rRNA gene sequences analysis of mucosal biopsies obtained during sigmoidoscopies from unprepared and prepared gut of the same individuals, it became clear that standard colonic lavage alters 
the composition and diversity of not only the intestinal lumen microbiota, but also the mucosa-associated, the differences being more prominent at the genus level [20]. It is now well accepted that the intestinal luminal and the mucosa-associated microbiota differ significantly from each other in diversity and composition, and appear as two distinct ecosystems with different metabolic and immunological functions [21].

Furthermore, when the intestinal microbiota composition was analyzed at baseline, immediately after bowel cleansing, and after 14 and 28 days, the number of bacteria in samples collected immediately after bowel cleansing was on average 34.7-fold lower than at baseline $(\mathrm{P}<0.001)$, and the number of methanogenic archaea was also decreased 20 -fold $(\mathrm{P}<0.001)$; these had returned to baseline by the 14 - and 28-day samples [22]. So far, it seems that bowel cleansing could be salutary for patients who are to undergo colon surgery. However, further analysis revealed that immediately after the lavage, the intestinal microbiota was significantly different from baseline, even at class or family level: there was a significant decrease in Bacilli and Clostridium cluster IV genera and a parallel significant increase in members of the Proteobacteria phylum and Clostridium cluster XIVa; additionally, the ratio of Gram-positive to Gram-negative species changed significantly after the lavage (from $5.3 \pm 4.8$ to $9.2 \pm 7.5$ at the 14 -day time-point, $\mathrm{P}<0.05$, after which a trend towards baseline was evidenced), while Proteus genera were still significantly increased after 28 days (Fig. 2).

In the same manner, a very recently published paper further underlines that, immediately after bowel lavage, a significant reduction in Lactobacillaceae and an increase in Enterobacteriaceae abundance were prominent; 30 days later these families were still significantly lower, while Streptococcaceae had increased 4-fold compared with samples collected before lavage [23].

These recent findings seem to provide clear evidence that the widely used polyethylene glycol bowel-cleansing preparation could be considered bacterial genocide, as it has a long-lasting effect on the composition and homeostasis of gut microbiota. It is well-known that laxatives in general introduce an osmotic flow of fluids into the gut, washing out the fecal luminal content with a substantial reduction in intestinal bacteria [24], while the concomitant rapid increase in gut motility further contributes

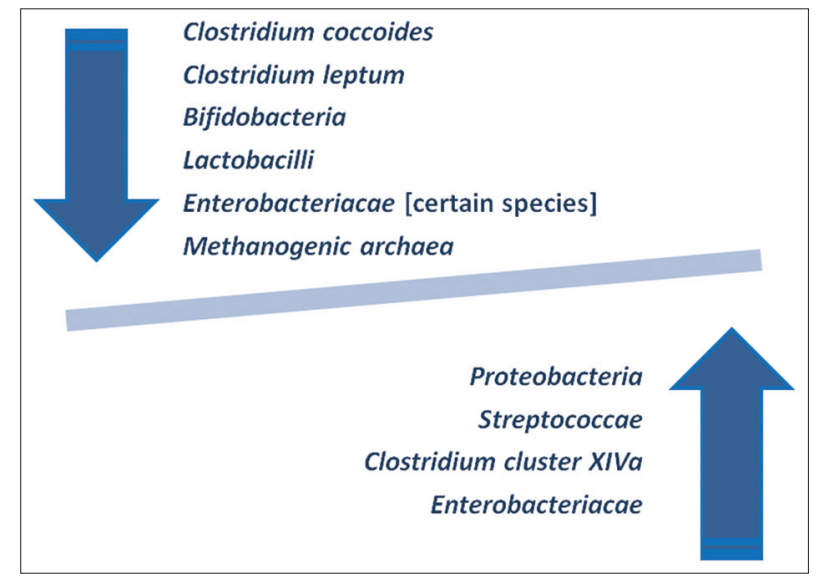

Figure 2 Alterations of the gut microbiome after mechanical bowel cleansing to flushing out all bacteria incapable of adhering to the gut mucosa, thus distorting the fecal bacterial composition [22,25].

Moreover, bowel purgation affects the quality and production of the protective mucus layer, while the fact that Proteobacteria flourish after lavage and in the long-term thereafter could be completely explained by the knowledge that purging leads to the introduction of oxygen into the normally anaerobic ecosystem and to an increase in $\mathrm{pH}$, via the loss of short-chain fatty acids $[25,26]$.

Finally, it has also been suggested that the sheer mechanical effect of colonic lavage may alter the intracellular signaling pathways involved in cell proliferation and influence the interaction between intestinal mucosal cells and the extracellular matrix, all of which are key elements of the mucosal gut barrier [27] (Fig. 3).

\section{Intestinal microbiome and antibiotics}

Antibiotic administration has long been known to have detrimental effects on the ecology of commensal bacteria, ranging from self-treated "functional" diarrhea to lifethreatening pseudomembranous colitis $[28,29]$. Recent studies have demonstrated that beyond the prolonged disruption of the intestinal microbial content at the taxa level, antibiotics also affect gene expression, protein activity and more than $87 \%$ of all metabolites, thus deranging the majority of metabolic pathways of critical importance to host physiology. They have also underlined that antibiotics lead to a significant alteration of the gut microbiome and a parallel decrease in microbial diversity of between one fourth and one third [12,30-32].

Today, it is more or less clear that even short-term antibiotic treatment can cause detrimental damage to the intestinal microbiome that can last more than 24 months. Panta et al [32] investigated the number and composition of the fecal microbiota just before and after a 7-day treatment in 21 patients who received fluoroquinolones, $\beta$-lactams and other commonly used antibiotics. Quantitative polymerase chain reaction analysis and pyrosequencing of the 16S rRNA gene reveal that both fluoroquinolones and $\beta$-lactams significantly decrease microbial diversity by $25 \%$, reducing the core phylogenetic taxa from 29 to only 12 . At the phylum level, both antibiotics resulted in a 2.5 -fold $(\mathrm{P}=0.0003)$ decrease in Firmicutes and an increase in Bacteroidetes, although this phenomenon was not prominent after treatment with piperacillin/tazobactam and levofloxacin/metronidazole.

Earlier studies showed that, during a 10-day amoxicillinclavulanic acid administration, Bifidobacterium spp. (one of the major groups seen on day 0 ) disappeared as early as day 4 , and had not returned by day 24 . In contrast, Enterobacteriaceae (which represented only $2 \%$ of the day 0 sequences) increased to $34 \%$ on day 4 , but were partially restored, as were the other major bacterial clusters, on day 24 [33]. Similarly, during a 5-day amoxicillin treatment, the dominant species presented on day 0 showed a major shift starting from day 1 , reaching an average similarity of only $74 \%$ after 4 days, after which they were partially restored to $88 \%$ on day 30 and to $89 \%$ only on day 60 [34]. 


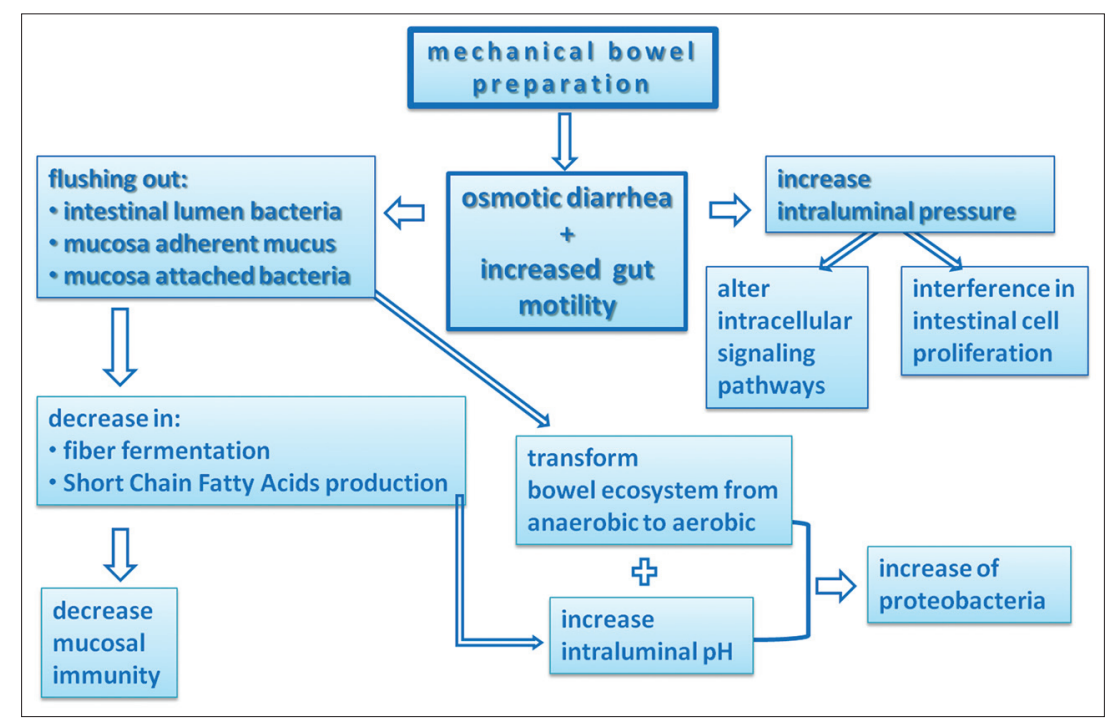

Figure 3 Effects of mechanical bowel cleansing on the intestinal microbiota

Finally, a 5-day ciprofloxacin administration was found to reduce the intestinal microbiota diversity, with significant effects on about one third of the bacterial taxa [31], the effects being less pronounced than those of clindamycin or amoxicillinclavulanic acid [35]. This taxonomic disturbance had recovered to almost the pre-treatment state at 4 weeks post-treatment, but several taxa failed to recover within 6 months [31].

\section{Other interventions}

Today, it is generally known that many of the infectious bacteria species acquire the capacity not only to recognize stress-related hormones, but also to synthesize the very same neurochemicals, which can influence the host. In other words, pathogenic bacteria in the stressed host may use stressreleased hormones as environmental cues by which to sense their surroundings $[36,37]$. It is also well known that microbes constantly assess their microenvironment and alter their phenotypic expression to optimize their survival, which means they tightly modify the expression of virulence in response to specific environmental cues $[4,10]$.

It has been shown that catecholamines directly affect the growth and expression of virulence-related factors in some bacteria, such as Yersinia enterocolitica, Escherichia coli (E. coli), Pseudomonas aeruginosa (P. aeruginosa), Salmonella typhii or Campylobacter jejuni [38,39]. Furthermore, there is evidence that the in-vitro growth of the respiratory pathogen Bordetella bronchiseptica (B. bronchiseptica) is greatly enhanced in the presence of norepinephrine and that this ability is, in part, mediated by the ability of norepinephrine to increase the acquisition of transferrin-bound iron by B. bronchiseptica [40]. In the same manner, norepinephrine was found to increase the proliferation of Streptococcus pneumoniae by assisting the delivery of iron from host iron-binding proteins, while at the same time enhancing the formation of biofilms and thus increasing antibiotic resistance [39].
Morphine is produced endogenously during the inflammatory processes by different cell types, including neutrophils, which rapidly transfer it to sites of inflammation and infection [41]. Additionally, morphine, one of the most commonly used analgesics, is considered a powerful immunosuppressant [42]; therefore, the sustained exposure of tissues to morphine, either endogenously produced or exogenously supplied, is a virtual certainty for all surgical patients, those with trauma, or the critically ill.

Morphine treatment in mice whose gut had been contaminated with $P$. aeruginosa caused a shift towards a more virulent phenotype of $P$. aeruginosa, able to cause lethal gut-derived sepsis, and a tendency for biofilm formation, thus increasing its antibiotic resistance. Moreover, $P$. aeruginosa possesses the ability to switch phenotype from being mucusenhancing to mucus-suppressing - having the ability to destroy gut epithelial integrity - depending on the presence or absence of morphine [43]. Additionally, Banerjee et al [44] have very recently shown for the first time that chronic morphine treatment significantly alters the gut microbiome composition and induces a preferential expansion of Grampositive as well as a reduction in bile-deconjugating bacterial strains.

Last but not least, food restriction, even in the setting of complete intravenous nutrition, leads to a scarcity of macronutrients for the bacteria within the gut, and thus to a relative loss of Firmicutes and to an expansion of Proteobacteria and Bacteroidetes. The hostile environment may favor Proteobacteria, because they have been shown to survive in states of relative starvation, versus Firmicutes, which dominate in a nutrient-rich environment $[45,46]$. Furthermore, a micronutrient insufficiency in the host, such as a lack of iron and phosphate, results in an analogously deprived environment, and it is well-known that local tissue phosphate concentration functions as an important cue through which endogenous bacteria "taste" the resources of the host to determine whether they should colonize or invade the host [4]. 


\section{Decreased microbial diversity, virulence and postoperative complications}

It is increasingly recognized that the gut microbiome plays a fundamental role in the health maintenance of the host, and that any alteration in the diversity, the number or the virulent phenotype can have a critical effect on host morbidity and even mortality. The concept that bacteria are able to sense the host environment, and adjust their behavior and virulence accordingly, is a new dimension in the area of intense research in severe-infection patients that breaks new ground in our understanding of how the gut acts as the driving force of critical illness [47]. Based on these observations, it is obvious that when, for whatever reason, the symbiotic relationship with the host is turned to dysbiosis, the newly pathogenic bacteria can further trigger and promote harm to the already compromised host, in a positive spiral feedback.

Since medical interventions and surgical manipulation of the host are part of everyday practice, it would be of great interest and importance to examine the precise mechanisms and correlate the reported alterations of the microbiome with the infectious complications in the surgical and/or critically ill patient. Shimizu et al $[3,13]$ found a significant reduction in the total anaerobic bacteria, as well as 2-log higher counts of the hazardous Staphylococcus and Pseudomonas groups, in the fecal flora of patients with SIRS, compared to healthy volunteers. Furthermore, they correlated key bacteria in the gut and derived their cutoff values in relation to infectious complications and mortality. The equilibrium between obligate anaerobes and total facultative anaerobes seems to play a critical role in causing septic complications: during the unfavorable evolution of SIRS, alterations in gut bacteria usually progress from a diverse pattern to a single pattern and then on to a depleted pattern, the three types representing a continuum of abnormality, depending on the severity of the patient's condition. Bacteremia was evident in 35\% of those with a diverse pattern versus $71 \%$ with the single pattern, resulting in a mortality rate of $6 \%$ in the former, $52 \%$ in the letter, and $64 \%$ in those with a depleted pattern $(\mathrm{P}<0.05)$ [48].

Liu et al [49] analyzed the feces of patients undergoing colorectal surgery and found a reduction in microbial diversity, including Bifidobacteria and Lactobacilli. In contrast, the numbers of Enterobacteriaceae, Pseudomonas and Candida, showed a significant increase, which in turn was well correlated with the higher rate of infectious complications, $46 \%$ versus $14 \%$, in probiotics-treated patients $(\mathrm{P}<0.05)$. Likewise, Komatsu [50] reported a significant reduction in the total number of bacteria and the number of dominant obligate anaerobes and a significant increase in the number of Enterobacteriaceae, Staphylococcus (MSCNS), Pseudomonas, and Clostridium difficile after colorectal surgery, compared with data from the same group before surgery.

Finally, in a recent study, neonatal piglets that underwent intestinal resection and received parenteral nutrition and antibiotics or placebo were examined at day 7 against agematched sow-fed piglets. Ileal and colonic contents revealed dramatic differences in diversity and an almost complete loss of Lactobacillus, along with a remarkable increase in the
Fusobacteriaceae and Enterobacteriaceae families in both the ileum and the colon. In addition, there was an increase in the Bacteroidaceae family in the colon [51]. These results strongly support similar findings in humans undergoing small bowel resection, who lacked exposure to enteral nutrition for 2 weeks. The reported loss in fecal bacterial diversity in this study was clearly associated with a higher incidence of postoperative infectious and anastomotic complications [52].

\section{Anastomotic leaks}

In colorectal surgery, an anastomotic leak represents the most dreaded of all complications, since it is often perceived as a failure of the operation or the surgeon, although the real cause of dehiscence is not fully elucidated. However, it has long been known that the intestinal bacterial population plays rather an important role: inoculation of rats with $10^{9} \mathrm{P}$. aeruginosa led to an increase in the incidence of anastomotic insufficiency up to $95 \%$ after gastrectomy and to a significant increase in mortality [53].

This concept has re-emerged as a result of advances in microbial isolation and identification using $16 \mathrm{~S}$ rRNA analysis. Olivas et al [54], working in an rat model of preoperative irradiation plus colonic resection and anastomosis, demonstrated that intestinal colonization with $P$. aeruginosa resulted in a significantly higher incidence of leaks, compared to the non-colonized group. What is even more striking is that the Pseudomonas colonizing anastomotic sites had become, in vivo, transformed to express a tissue-destroying phenotype; that is, one that had undergone a single nucleotide polymorphism mutation in the mexT gene that resulted in a much more virulent phenotype with increased collagenase activity, high swarming motility, and an increased ability for tissue destruction.

It is well known that important human mucosal pathogens have evolved virulence mechanisms to circumvent the mucosal epithelium barrier [55,56]. P. aeruginosa seems to favor damaged epithelial tissues to initiate colonization [57]; then, upon binding to epithelial cells, it activates a phosphatidylinositol 3-kinase, which is absolutely necessary for $P$. aeruginosa to enter from the apical surface of polarized epithelial cells, by subverting the epithelial cell polarity [56].

Further studies have demonstrated that the anastomosis construction itself causes significant alterations to the bacterial composition at the anastomotic site, but not to the luminal microbial content [58]. The most interesting observations are that the Enterococcus and the Escherichial Shigella populations increased by 500 -fold and 200 -fold, respectively; at the same time, populations of beneficial bacteria were reduced $[59,60]$. For an in-depth analysis of the marked Enterococcus increase [58] and the associated high collagen-degrading activity, they inoculated Enterococcus faecalis (E. faecalis) strains obtained just after completion of the colorectal anastomosis in a rat model and on the sixth postoperative day; by collecting the liquids drained from the gut, they demonstrated that the collagen-degrading activity of the bacteria recovered from the anastomotic area enabled 
discrimination between leaking and non-leaking anastomotic sites. They also found that E. faecalis exhibited an increased ability to activate tissue matrix metalloproteinase-9, operated through the gelE and $\operatorname{sprE}$ genes, both of which contributed to anastomotic leakage [61].

The microbiome of eight patients who experienced colorectal anastomotic rupture and of another eight matched for age, gender and adjuvant therapy, was investigated by studying the rings of colon and rectum tissues cut by the circular stapler to make the anastomosis [62]. The investigators surprisingly reported a significantly higher proportion of the Lachnospiraceae family versus controls-although these bacteria tend to be rather friendly to the bowel, as most of them belong to butyrate producing genera. However, further analysis revealed that a large fraction of the Lachnospiraceae were identified to be of the mucin-degrading Ruminococcus, and that Lachnospiraceae levels were strongly negatively correlated with microbial diversity levels, which in turn are associated with anastomotic leakage.

\section{Probiotics - prebiotics - synbiotics}

Probiotics are live microbial food supplements that may beneficially affect the host by improving its intestinal microbial balance, while prebiotics are indigestible fibers that promote the growth and function of probiotics; their combination is called synbiotics [7]. Probiotics are able to maintain gut barrier function by restoring intestinal permeability and ameliorating the intestinal inflammatory response and the release of cytokines, and can also maintain the homeostasis of the normal gut microbiota. As a result, they have been extensively studied as an adjuvant perioperative treatment modality for surgical patients $[7,11]$. In the field of gastrointestinal surgery, it has been shown that probiotics may be effective in restoring gut microbiota diversity, enhancing immunological response, reducing the systemic inflammatory response released postoperatively, and improving patients' quality of life. Moreover, as a consequence of all the above, they appear to work positively in reducing the total length of hospital stay, the number of days of ventilator support required and of days in intensive care, and the overall infectious complications [63-66]. However, other investigators have reported no benefits after the perioperative use of probiotics in patients undergoing elective abdominal surgery. A possible explanation of these differences may be related to the rather short administration period (median of 4 days) in the majority of the studies, the low concentration of bacteria present in the formulation prescribed, the one probiotic strain only of the regimen used, and the small number of participants in most studies. Last but not least, consideration must be given to the open-gut manipulation strategies applied, which fortify the bacterial contamination of the peritoneal cavity and the interruption of blood supply to the viscera, due both to the ligation of major vessels and the use of heat-coagulation for the smaller ones $[67,68]$. Finally, many clinicians start with the negative assumption that, given the degree of diversity and metabolic functions of the normal core microbiome, it appears naïve to believe that some Lactobacilli strains could fully supplant the degree of functionality required of the intestinal microbiome to bolster systemic immune function during disease states.

\section{Probiotics and infectious complications}

Multiple studies have been performed regarding the potential benefit of enteral administration of probiotics in reducing infectious complications in surgical as well as in critically ill patients, based on the idea that they may modify the gastrointestinal bacteria in a manner that preferentially favors the growth of minimally virulent species [69]. He et al [68] analyzed six randomized controlled trials dealing with pro/synbiotic administration in 361 colon cancer patients undergoing colorectal resection and found a significant decrease in the total number of infections $(\mathrm{P}=0.001)$, mainly due to the decreased cases of pneumonia $(\mathrm{P}=0.04)$, while other infectious complications, such as surgical site or intraabdominal infections or bacteremia, remained unaffected.

This is in line with a previous meta-analysis that demonstrated a significant reduction in the rate of nosocomial pneumonia $(\mathrm{P}=0.03)$ in critically ill patients treated with probiotics [70], as was also reported in relation to ventilatoracquired pneumonia (VAP) [71]. Various other studies have demonstrated similar effects, while a recent meta-analysis suggests that probiotic treatment results in a 39\% reduction in VAP, along with a subsequent reduction in the length of ICU stay [72]. A possible mode of action is considered to be the ability of probiotics to inhibit or ameliorate gastrointestinal and systemic bacterial colonization [73], since it has been shown that probiotic-treated patients exhibit smaller rates of $P$. aeruginosa colonization versus controls [72]. In a metaanalysis of 5 trials (844 patients), probiotics showed a trend towards a lower incidence of VAP; when one trial was excluded, a statistically significant conclusion could be drawn. Thus, the administration of probiotics seems to significantly reduce the risk of VAP caused by P. aeruginosa [74].

Another study suggested that modification of the upper aerodigestive flora by means of applying probiotics could reduce nosocomial infections [69]; in any case, Lactobacillus administration resulted in a significant delay in the time to onset of VAP $(\mathrm{P}<0.001)$, as has also been documented in a study of our group, where a statistically significant delay in the time of blood infection onset was prominent [75].

In a randomized controlled study of colorectal cancer patients, Zhang et al [64] demonstrated a significant reduction in septic complications $(33.3 \%$ in controls versus $10 \%$ in probiotic-treated), along with a significant decrease in E. coli and a significant increase in the Bifidobacterium counts in the same group. Likewise, fecal cultures in ICU multiple trauma patients, symbiotic-treated, revealed a decrease in Enterobacteriaceae, coagulase-negative Staphylococci, and Gram-negative anaerobes, and an increase in Enterococcus spp. and Gram-positive anaerobes [76]. These patients demonstrated a $13.9 \%$ incidence of bacteremia versus $36.1 \%$ in those receiving placebo $(\mathrm{P}=0.028)$, a finding 
related to the reduced incidence of Acinetobacter baumaniirelated, ventilator-associated pneumonia [77]. Various other studies have produced similar results, with Liu [78], in a study of colorectal cancer patients, reporting rates of bacteremia of $55 \%$ in the probiotics group versus $72 \%$ in controls, $\mathrm{P}=0.017$.

Furthermore, a recent meta-analysis demonstrated a reduction of postoperative sepsis after elective general surgery, both in pro- and synbiotic-treated patients compared to placebo ( $\mathrm{P}=0.003$ and 0.002 , respectively). However, no significant difference in the incidence of pneumonia, urinary tract or surgical site infections was found [79], while a very recently published study revealed a significant decrease of $38 \%$ in the incidence of postoperative sepsis. Separate analysis according to the type of operation revealed a statistically significant difference among the types, with a $35 \%$ risk reduction in colorectal surgery, $73 \%$ in hepato-pancreatico-biliary, and a $56 \%$ risk reduction in liver transplant operations; these findings add to the evidence that colorectal surgery patients might be the most difficult group for the manipulation of gut microbial balance [80].

Finally, in a recently conducted systematic review, the mean incidence of surgical site infection was $6.8 \%$ in treated patients and $11.1 \%$ in controls, representing a $37 \%$ reduction. This study also underlined the potential benefit in relation to urinary tract infections and composite infections, as well as the non-occurrence of serious adverse events related to study products [81].

Moreover, it is now common knowledge that, because of the complexity of the individual gut microbiome, probiotics are not a one-species-fits-all approach [82]; thus, when a single probiotic regimen (Lactobacillus plantarum [L. plantarum] 299v) was used in patients undergoing colectomy, no benefit was found regarding postoperative complications [67].

\section{Probiotics and colon anastomosis failure}

Taking into account the further progress in the research of Shogan et al [58], demonstrating that anastomosis construction itself causes significant alterations to the bacterial composition at the anastomotic site, and the recent knowledge that all medical and surgical interventions related to anastomosis construction contribute to a reduction in bacteria diversity, including the eradication of beneficial and the increasing virulence of noxious species [54,61], it is reasonable to seriously reconsider the crucial role of bacteria in undermining the healing process and to look for a mode of enrichment of the scarce or destroyed bacterial species, the simplest and easiest mode being probiotics.

The first single-center randomized clinical study to evaluate the effect of probiotic treatment on the incidence of colon anastomotic failure was that of Zhang et al [64]. They demonstrated only a slight reduction in the rate of anastomotic leaks $(0 / 30$ in probiotics-treated individuals versus $2 / 30$ controls $(\mathrm{P}=0.49)$. However, the patients had received 3 oral viable capsules/day for 3 days only $\left(10^{8} \mathrm{cfu} / \mathrm{g}\right.$ of Bifidobacterium longum, Lactobacillus acidophilus [L. acidophilus], and E. faecalis), from day -5 to day -3 , followed by conventional bowel preparation plus oral gentamicin for 3 days.

In a recent randomized study by our group, involving patients undergoing colorectal surgery for cancer, a four-probiotic formulation (L. acidophilus, L. plantarum, Bifidobacterium lactis and Saccharomyces boulardii) or placebo was administered, starting one day before surgery - after mechanical bowel cleansing - and continuing for 15 days postoperatively, the patients being followed-up for complications for 30 days. The probiotic-treated group exhibited a significantly lower rate of all postoperative major complications (28.6\% versus $48.8 \%$ in the placebo arm, $\mathrm{P}=0.010)$, postoperative pneumonia $(2.4 \%$ versus $11.3 \%, \mathrm{P}=0.029)$, surgical site infections ( $7.1 \%$ versus $20.0 \%, \mathrm{P}=0.020)$, and anastomotic leakage $(1.2 \%$ vs. $8.8 \%$, $\mathrm{P}=0.031)[63]$.

Moreover, after total RNA extraction, it was also clearly found that in probiotics versus controls, there was modulation of suppressor of cytokine stimulation-3 (SOCS3) expression which encodes for the protein SOCS3 that finally suppresses overwhelming cytokine responses. In other words, the prophylactic action of probiotics in these colon-cancer patients is exerted through modulation of the immune response and is linked with the prevention of immunosuppression development after a bacterial challenge [64].

From all the above analyses, we would summarize that exogenously given probiotics contribute at least partially to the restoration of the decreased gut microbial diversity, but mainly preserve the host's immune function; i.e., they prevent immunosuppression, which might otherwise be "detected" by the pathogens and trigger changes in their virulence and lethality as they then attack the host.

\section{Concluding remarks}

Modulation of the intestinal microbiota with probiotics seems to be an effective method of reducing infectious complications in surgical patients, although their effect on mortality has still not been elucidated. Further studies need to be conducted to establish the best possible combination of probiotics, as well as to determine the subgroup of patients who could benefit most from such an intervention.

\section{References}

1. Alverdy J, Holbrook C, Rocha F, et al. Gut-derived sepsis occurs when the right pathogen with the right virulence genes meets the right host: evidence for in vivo virulence expression in Pseudomonas aeruginosa. Ann Surg 2000;232:480-489.

2. Rasid O, Cavaillon JM. Recent developments in severe sepsis research: from bench to bedside and back. Future Microbiol 2016;11:293-314.

3. Shimizu K, Ogura H, Hamasaki T, et al. Altered gut flora are 
associated with septic complications and death in critically ill patients with systemic inflammatory response syndrome. Dig Dis Sci 2011;56:1171-1177.

4. Morowitz MJ, Babrowski T, Carlisle EM, et al. The human microbiome and surgical disease. Ann Surg 2011;253:1094-1101.

5. Zaborin A, Smith D, Garfield K, et al. Membership and behavior of ultra-low-diversity pathogen communities present in the gut of humans during prolonged critical illness. MBio 2014;5:e1361-e01414.

6. Claesson MJ, Jeffery IB, Conde $S$, et al. Gut microbiota composition correlates with diet and health in the elderly. Nature 2012;488:178-184.

7. Kotzampassi K, Giamarellos-Bourboulis EJ. Probiotics for infectious diseases: more drugs, less dietary supplementation. Int J Antimicrob Agents 2012;40:288-296.

8. Khanna S, Tosh PK. "A clinician's primer on the role of the microbiome in human health and disease. Mayo Clin Proc 2014;89:107-114.

9. Qin J, Li R, Raes J, et al. A human gut microbial gene catalogue established by metagenomic sequencing. Nature 2010;464:59-65.

10. Eckburg PB, Bik EM, Bernstein CN, et al. Diversity of the human intestinal microbial flora. Science 2005;308:1635-1638.

11. Linares DM, Ross P, Stanton C. Beneficial microbes: The pharmacy in the gut. Bioengineered 2016;7:11-20.

12. Francino MP. Antibiotics and the human gut microbiome: dysbioses and accumulation of resistances. Front Microbiol 2016;6:1543.

13. Shimizu K, Ogura $\mathrm{H}$, Goto $M$, et al. Altered gut flora and environment in patients with severe SIRS. J Trauma 2006;60:126-133.

14. Eleftheriadis E, Kotzampassi K, Papanotas K, Heliadis N, Sarris K. Gut ischemia, oxidative stress, and bacterial translocation in elevated abdominal pressure in rats. World J Surg 1996;20:11-16.

15. Lapthorne S, Bines JE, Fouhy F, et al. Changes in the colon microbiota and intestinal cytokine gene expression following minimal intestinal surgery. World $J$ Gastroenterol 2015;21:4150-4158.

16. Watanabe M, Murakami M, Nakao K, Asahara T, Nomoto K, Tsunoda A. Randomized clinical trial of the influence of mechanical bowel preparation on faecal microflora in patients undergoing colonic cancer resection. Br J Surg 2010;97:1791-1797.

17. Fry DE. Colon preparation and surgical site infection. Am J Surg 2011;202:225-232.

18. Slim K, Vicaut E, Launay-Savary MV, Contant C, Chipponi J. Updated systematic review and meta-analysis of randomized clinical trials on the role of mechanical bowel preparation before colorectal surgery. Ann Surg 2009;249:203-209.

19. Güenaga KF, Matos D, Wille-Jørgensen P. Mechanical bowel preparation for elective colorectal surgery. Cochrane Database Syst Rev 2011;9:CD001544.

20. Harrell L, Wang Y, Antonopoulos D, et al. Standard colonic lavage alters the natural state of mucosal-associated microbiota in the human colon. PLoS One 2012;7:e32545.

21. Ringel Y, Maharshak N, Ringel-Kulka T, Wolber EA, Sartor RB, Carroll IM. High throughput sequencing reveals distinct microbial populations within the mucosal and luminal niches in healthy individuals. Gut Microbes 2015;6:173-181.

22. Jalanka J, Salonen A, Salojärvi J, et al. Effects of bowel cleansing on the intestinal microbiota. Gut 2015;64:1562-1568.

23. Drago L, Toscano M, De Grandi R, Casini V, Pace F. Persisting changes of intestinal microbiota after bowel lavage and colonoscopy. Eur J Gastroenterol Hepatol 2016;28:532-537.

24. Wexner SD, Beck DE, Baron TH, et al. A consensus document on bowel preparation before colonoscopy: prepared by a task force from the American Society of Colon and Rectal Surgeons (ASCRS), the American Society for Gastrointestinal Endoscopy (ASGE), and the Society of American Gastrointestinal and Endoscopic Surgeons (SAGES). Gastrointest Endosc 2006;63:894-909.

25. Strocchi A, Bond JH, Ellis C, Levitt MD. Colonic concentrations of hydrogen and methane following colonoscopic preparation with an oral lavage solution. Gastrointest Endosc 1990;36:580-582.

26. Johansson ME, Gustafsson JK, Holmén-Larsson J, et al. Bacteria penetrate the normally impenetrable inner colon mucus layer in both murine colitis models and patients with ulcerative colitis. Gut 2014;63:281-291.

27. Gayer CP, Basson MD. The effects of mechanical forces on intestinal physiology and pathology. Cell Signal 2009;21:1237-1244.

28. Cotter PD, Stanton C, Ross RP, Hill C. The impact of antibiotics on the gut microbiota as revealed by high throughput DNA sequencing. Discov Med 2012;13:193-199.

29. Macfarlane S. Antibiotic treatments and microbes in the gut. Environ Microbiol 2014;16:919-924.

30. Antunes LC, Han J, Ferreira RB, Lolić P, Borchers CH, Finlay BB. Effect of antibiotic treatment on the intestinal metabolome. Antimicrob Agents Chemother 2011;55:1494-1503.

31. Dethlefsen L, Huse S, Sogin ML, Relman DA. The pervasive effects of an antibiotic on the human gut microbiota, as revealed by deep 16S rRNA sequencing. PLoS Biol 2008;6:e280.

32. Panda S, El khader I, Casellas F, et al. Short-term effect of antibiotics on human gut microbiota. PLoS One 2014;9:e95476.

33. Young VB, Schmidt TM. Antibiotic-associated diarrhea accompanied by large-scale alterations in the composition of the fecal microbiota. J Clin Microbiol 2004;42:1203-1206.

34. De La Cochetière MF, Durand T, Lepage P, Bourreille A, Galmiche JP, Doré J. Resilience of the dominant human fecal microbiota upon short-course antibiotic challenge. J Clin Microbiol 2005;43:5588-5592.

35. Donskey CJ, Hujer AM, Das SM, Pultz NJ, Bonomo RA, Rice LB. Use of denaturing gradient gel electrophoresis for analysis of the stool microbiota of hospitalized patients. J Microbiol Methods 2003;54:249-256.

36. Asano Y, Hiramoto T, Nishino R, et al. Critical role of gut microbiota in the production of biologically active, free catecholamines in the gut lumen of mice. Am J Physiol Gastrointest Liver Physiol 2012;303:G1288-G1295.

37. Lyte M. The effect of stress on microbial growth. Anim Health Res Rev 2014; 15:172-174.

38. Konturek PC, Brzozowski T, Konturek SJ. Stress and the gut: pathophysiology, clinical consequences, diagnostic approach and treatment options. J Physiol Pharmacol 2011;62:591-599.

39. Sandrini S, Alghofaili F, Freestone P, Yesilkaya H. Host stress hormone norepinephrine stimulates pneumococcal growth, biofilm formation and virulence gene expression. BMC Microbiol 2014;14:180.

40. Anderson MT, Armstrong SK. Norepinephrine mediates acquisition of transferrin-iron in Bordetella bronchiseptica. J Bacteriol 2008;190:3940-3947.

41. Glattard E, Welters ID, Lavaux T, et al. Endogenous morphine levels are increased in sepsis: a partial implication of neutrophils. PLoS One 2010;5:e8791.

42. Ma J, Wang J, Wan J, et al. Morphine disrupts interleukin-23 (IL-23)/IL-17-mediated pulmonary mucosal host defense against Streptococcus pneumoniae infection. Infect Immun 2010;78:830-837.

43. Babrowski T, Holbrook C, Moss J, et al. Pseudomonas aeruginosa virulence expression is directly activated by morphine and is capable of causing lethal gut-derived sepsis in mice during chronic morphine administration. Ann Surg 2012;255:386-393.

44. Banerjee S, Sindberg G, Wang F, et al. Opioid-induced gut microbial disruption and bile dysregulation leads to gut barrier compromise and sustained systemic inflammation. Mucosal Immunol 
2016 Feb 24. doi: 10.1038/mi.2016.9. [Epub ahead of print].

45. Demehri FR, Barrett M, Teitelbaum DH. Changes to the intestinal microbiome with parenteral nutrition: review of a murine model and potential clinical implications. Nutr Clin Pract 2015;30:798-806.

46. Mountzouris KC, Kotzampassi K, Tsirtsikos P, Kapoutzis K, Fegeros K. Effects of Lactobacillus acidophilus on gut microflora metabolic biomarkers in fed and fasted rats. Clin Nutr 2009;28:318-324.

47. Swank GM, Deitch EA. Role of the gut in multiple organ failure: bacterial translocation and permeability changes. World J Surg 1996;20:411-417.

48. Shimizu K, Ogura H, Tomono K, et al. Patterns of Gram-stained fecal flora as a quick diagnostic marker in patients with severe SIRS. Dig Dis Sci 2011;56:1782-1788.

49. Liu Z, Qin H, Yang Z, et al. Randomised clinical trial: the effects of perioperative probiotic treatment on barrier function and postoperative infectious complications in colorectal cancer surgery - a double-blind study. Aliment Pharmacol Ther 2011;33:50-63.

50. Komatsu S, Sakamoto E, Norimizu S, et al. Efficacy of perioperative synbiotics treatment for the prevention of surgical site infection after laparoscopic colorectal surgery: a randomized controlled trial. Surg Today 2016;46:479-490.

51. Levesque CL, Turner J, Li J, et al. In a neonatal piglet model of intestinal failure, administration of antibiotics and lack of enteral nutrition have a greater impact on intestinal microflora than surgical resection alone. JPEN J Parenter Enteral Nutr 2016 Feb 2. pii: 0148607115626903 . [Epub ahead of print].

52. Ralls MW, Miyasaka E, Teitelbaum DH. Intestinal microbial diversity and perioperative complications. JPEN J Parenter Enteral Nutr 2014;38:392-399.

53. Schardey HM, Kamps T, Rau HG, Gatermann S, Baretton G, Schildberg FW. Bacteria: a major pathogenic factor for anastomotic insufficiency. Antimicrob Agents Chemother 1994;38:2564-2567.

54. Olivas AD, Shogan BD, Valuckaite V, et al. Intestinal tissues induce an SNP mutation in Pseudomonas aeruginosa that enhances its virulence: possible role in anastomotic leak. PLoS One 2012;7:e44326.

55. Kazmierczak BI, Mostov K, Engel JN. Interaction of bacterial pathogens with polarized epithelium. Annu Rev Microbiol 2001;55:407-435.

56. Engel J, Eran Y. Subversion of mucosal barrier polarity by Pseudomonas aeruginosa. Front Microbio 2011;2:114.

57. Engel J, Balachandran P. Role of Pseudomonas aeruginosa type III effectors in disease. Curr Opin Microbiol 2009;12:61-66.

58. Shogan BD, Smith DP, Christley S, Gilbert JA, Zaborina O, Alverdy JC. Intestinal anastomotic injury alters spatially defined microbiome composition and function. Microbiome 2014;2:35.

59. Mack DR, Ahrne S, Hyde L, Wei S, Hollingsworth MA. Extracellular MUC3 mucin secretion follows adherence of Lactobacillus strains to intestinal epithelial cells in vitro. Gut 2003;52:827-833.

60. Okada M, Bothin C, Kanazawa K, Midtvedt T. Experimental study of the influence of intestinal flora on the healing of intestinal anastomoses. Br J Surg 1999;86:961-965.

61. Shogan BD, Belogortseva N, Luong PM, et al. Collagen degradation and MMP9 activation by Enterococcus faecalis contribute to intestinal anastomotic leak. Sci Transl Med 2015;7:286ra68.

62. van Praagh JB, de Goffau MC, Bakker IS, Harmsen HJ, Olinga P, Havenga K. Intestinal microbiota and anastomotic leakage of stapled colorectal anastomoses: a pilot study. Surg Endosc 2016;30:2259-2265.

63. Kotzampassi K, Stavrou G, Damoraki G, et al. A four-probiotics regimen reduces postoperative complications after colorectal surgery: a randomized, double-blind, placebo-controlled study. World J Surg 2015;39:2776-2783.

64. Zhang JW, Du P, Gao J, Yang BR, Fang WJ, Ying CM. Preoperative probiotics decrease postoperative infectious complications of colorectal cancer. Am J Med Sci 2012;343:199-205.

65. Kotzampassi K, Eleftheriadis E. Synbiotics in trauma: of proven benefit or a new fad. In: Intensive Care Units: Stress, Procedures and Mortality Rates. Nova Science Publishers; 2011, pp. 149-158.

66. Stavrou G, Giamarellos-Bourboulis EJ, Kotzampassi K. The role of probiotics in the prevention of severe infections following abdominal surgery. Int J Antimicrob Agents 2015;46(Suppl 1):S2-S4.

67. Mangell P, Thorlacius H, Syk I, et al. Lactobacillus plantarum 299v does not reduce enteric bacteria or bacterial translocation in patients undergoing colon resection. Dig Dis Sci 2012;57:1915-1924.

68. He D, Wang HY, Feng JY, Zhang MM, Zhou Y, Wu XT. Use of pro-/synbiotics as prophylaxis in patients undergoing colorectal resection for cancer: a meta-analysis of randomized controlled trials. Clin Res Hepatol Gastroenterol 2013;37:406-415.

69. Morrow LE, Kollef MH, Casale TB. Probiotic prophylaxis of ventilator-associated pneumonia: a blinded, randomized, controlled trial. Am J Respir Crit Care Med 2010;182:1058-1064.

70. Liu KX, Zhu YG, Zhang J, et al. 'Probiotics' effects on the incidence of nosocomial pneumonia in critically ill patients: a systematic review and meta-analysis. Crit Care 2012;16:R109.

71. Bo L, Li J, Tao T, et al. Probiotics for preventing ventilator-associated pneumonia. Cochrane Database Syst Rev 2014;10:CD009066.

72. Siempos II, Ntaidou TK, Falagas ME. Impact of the administration of probiotics on the incidence of ventilator-associated pneumonia: a meta-analysis of randomized controlled trials. Crit Care Med 2010;38:954-962.

73. Barraud D, Bollaert PE, Gibot S. Impact of the administration of probiotics on mortality in critically ill adult patients: a metaanalysis of randomized controlled trials. Chest 2013;143:646-655.

74. Wang J, Liu KX, Ariani F, Tao LL, Zhang J, Qu JM. Probiotics for preventing ventilator-associated pneumonia: a systematic review and meta-analysis of high-quality randomized controlled trials. PLoS One 2013;8:e83934.

75. Giamarellos-Bourboulis EJ, Bengmark S, Kanellakopoulou K, Kotzampassi K. Pro- and synbiotics to control inflammation and infection in patients with multiple injuries. J Trauma 2009;67:815-821.

76. Koutelidakis IM, Bezirtzoglou E, Giamarellos-Bourboulis EJ, Grosomanidis V, Kotzampassi K. Impact of synbiotics on the intestinal flora of critically ill patients with multiple injuries. Int $J$ Antimicrob Agents 2010;36:90-91.

77. Kotzampassi K, Giamarellos-Bourboulis EJ, Voudouris A, Kazamias P, Eleftheriadis E. Benefits of a synbiotic formula (Synbiotic 2000Forte) in critically Ill trauma patients: early results of a randomized controlled trial. World J Surg 2006;30:1848-1855.

78. Liu ZH, Huang MJ, Zhang XW, et al. The effects of perioperative probiotic treatment on serum zonulin concentration and subsequent postoperative infectious complications after colorectal cancer surgery: a double-center and double-blind randomized clinical trial. Am J Clin Nutr 2013;97:117-126.

79. Kinross JM, Markar S, Karthikesalingam A, et al. A meta-analysis of probiotic and synbiotic use in elective surgery: does nutrition modulation of the gut microbiome improve clinical outcome? JPEN J Parenter Enteral Nutr 2013;37:243-253.

80. Arumugam S, Lau CS, Chamberlain RS. Probiotics and synbiotics decrease postoperative sepsis in elective gastrointestinal surgical patients: a meta-analysis. J Gastrointest Surg 2016;20:1123-1131.

81. Lytvyn L, Quach K, Banfield L, Johnston BC, Mertz D. Probiotics and synbiotics for the prevention of postoperative infections following abdominal surgery: a systematic review and meta-analysis of randomized controlled trials. J Hosp Infect 2016;92:130-139.

82. Tappenden KA. Probiotics are not a one-species-fits-all proposition. JPEN J Parenter Enteral Nutr 2012;36:496. 